\title{
The Trend of an Embedded Undersized Range Wireless Communication Technologies
}

\author{
Aiman J. Albarakati, \\ Department of Computer Engineering, \\ College of Computer and Information Science, \\ Majmaah University, \\ Majmaah, Saudi Arabia
}

\begin{abstract}
:
The trend of the up-to-date wireless technologies and embedded systems give the impression ordained to construct an outsized with enduring brunt and changes in our lives. It above all has been far and wide brought into play in the past and up to date expansions and growth in such technology endow with a new-fangled strait for realization of these systems with a great remote access. Currently, we do have a lot of wireless technologies with their individual distinct and nuisance. In view of that fact as embedded systems is application specific, for such reason it won't be suitable for other. Here in this article we have detailed such numerous restricted ranges wireless technologies.
\end{abstract}

\section{Keywords:}

Sensor Networks, Embedded Systems, WSN

\section{INTRODUCTION:}

Embedded Systems having dedicated programming design are normally associated with larger systems. They can be usually differing from desktop systems in regards of functionality/recitation. Embedded systems are preprogrammed and application restricted systems as compared to desktop systems which are all-purpose and reprogrammable systems. Almost each and every electronic device planned and manufactured in the present day is socalled embedded system, no matter whether it represents user, household, industrial, medicinal or military product/artifact. Intricacy in devise fluctuate from squat, with a solitary microcontroller chip, to an extremely towering with manifold units/components, networks and peripherals [1]

[2]. Loads of embedded system applications call for improvement in regards of interoperability, better coverage, entrée and data sharing and on hand possessions/services. In this perspective wireless utilization is one of the dominant ways out for offering better deployment of sovereignty and expediency. In most cases, such systems with capacity of sharing on hand data/services wirelessly will be extremely efficient and will unravel many areas for bringing them into practice [3][4].

\section{SHORT-RANGE TECHNOLOGY}

At present we have numerous wireless schemes for shortrange communication with an ambition of endowing a wireless connectivity. Several of such schemes are either standards or either proprietary. Technologies that are proprietary usage have the concerns of inter-operability as compared to the standardized scheme where no such issue arises. We will outline such technologies in our subsequent sections to give a detailed picture.

\subsection{Bluetooth:}

A high-speed, low-power microwave wireless link technology, projected to disseminate data over undersized detachments/distances from permanent/fixed and mobile devices, by fashioning personal area networks (PANs). It is Omni-directional and does not call for line-of-sight settings of associated devices. When a device having Bluetooth approach inside the range of another device having the same, then they right away swap over address information and generate minute ad-hoc networks known as "piconet" devoid of client participation. These networks are launched vigorously and routinely as Bluetooth enabled devices go through and depart radio immediacy. Following are the Bluetooth most important facets:

i. A global licensed band " $2.4 \mathrm{GHz}$ ISM (IndustrialScientific-Medical)" is used to operate them.

ii. It utilizes Frequency Hop Spread Spectrum (FHSS) technique.

iii. It utilizes Gaussian frequency-shift keying (GFSK) modulation technique.

iv. Have the capacity to prop or hold up to seven other devices in its established network.

v. It is Omni-directional means no line-of-sight settings is required.

vi. Power restricted/dependent range: Class $1-100 \mathrm{~mW}$ power, 100 meters range; Class $2-2.5 \mathrm{~mW}$ power, 10 meters range; Class $3-1 \mathrm{~mW}$ power, 1 meter range.

vii. It is an in-expensive technology and chip sets for put into practice Bluetooth are willingly offered.

viii. Unusual data rates as per version: Version 1.2 - Up to $1 \mathrm{Mbps}$; Version $2.0+\mathrm{EDR}$ - Up to $3 \mathrm{Mbps}$; Version 3.0 + HS - Up to 24 Mbps .

Bluetooth is capable enough to handle data and voice communication concurrently at the same time. Nevertheless, for the reason that Bluetooth-enabled devices be capable of communicating with a lot of devices straight away and with no acquaintance of the listener and its objective, data protection turns out to be an imperative reflection [5][6].

\subsection{Infrared Data Association (IrDA):}

This technology is an industry-based group of device manufacturers came into existence in late 1993 that constructed a standard for sending out data by means of infrared light waves. Infrared transmissions are intrinsically confined to a small area and regimes do not control the infrared fraction of the light spectrum. This standard or design is in particular appropriate for in-expensive, undersize and point-to-point (P2P) communication and is utilized for endowing wireless connectivity for devices that by and large would make use of a cable tilting elucidation. It endows with a set of protocols together with all layers of data transport, 
likewise a number of network administration qualifications and interoperability facets. Following are the IrDA most important facets:

i. Maneuver inside a range of at least 1 meter that have the capacity of further be extended to 2 meters.

ii. Power efficient and utilizes less power.

iii. Bi-directional or 2 way Communication.

iv. Having data rates starting from $9600 \mathrm{~b} / \mathrm{s}$ with prime speed/cost and steps of $115 \mathrm{~kb} / \mathrm{s}$ and utmost speed up to $4 \mathrm{Mb} / \mathrm{s}$.

v. Cycle Redundancy Check (CRC) is utilized to sheltered the data packets.

We don't have any integrated encryption technique in this standard. Since infrared signal that is send out in a constricted shaft region with extremely minute signal potency so the transmitter ought to be shut and aiming at the receiver. By having so there is no such real chance of eavesdropping or snooping [7].

\subsection{IEEE 802.11 standard:}

This standard has been designed by the IEEE(Institute of Electrical and Electronics Engineers) having the aptitude of carrying out wireless local area network (WLAN) computer communication/transmission in the $2.4,3.6$ and $5 \mathrm{GHz}$ frequency bands with Carrier Sense Multiple Access with Collision Avoidance (CSMA/CA) for path sharing. Phaseshift keying (PSK) was the imaginative modulation utilized in 802.11. Nevertheless, other methods, e.g. complementary code keying (CCK) are brought into play in a number of latest stipulations. The newer/latest modulation methods/schemes endow with privileged data alacrity/pace and concentrated weakness to intrusion. This is utilized to join two comparatively hefty devices that have heaps of power at towering speeds/pace. This expertise is also constructive to pull out LAN to positions or places where it is either costly or problematic to jog cables. Following are the IEEE 802.11 (WLAN) most important facets:

i. This standard brings into play the Direct-sequence spread spectrum (DSSS), Frequency hopping spread spectrum (FSSS), Orthogonal frequency division multiplexing (OFDM) modulation technique with Complementary code keying (CCK).

ii. It puts together $2.4 \mathrm{GHz}$ (ISM Band) and $5 \mathrm{GHz}$ frequency band.

iii. It endows with data rates of $1 \mathrm{Mbps}, 2 \mathrm{Mbps}, 11$ Mbps (802.11b), 54 Mbps (802.11a).

iv. It is utilized in a point-to-multipoint configuration.

v. It has an Operating range up to 155 feet indoors and 1500 feet outdoors.

$\mathrm{Wi}-\mathrm{Fi}$ is the up-to-the-minute name agreed to products incorporating the IEEE $802.11 \mathrm{a} / \mathrm{b} / \mathrm{g}$ local-area network (LAN) archetype. This has turn out to be one of the flourishing shortrange/undersized wireless technology in concerning laptops, institutions, office or home local-area networks. And data security is the crucial concern for such technology. In the beginning Wired Equivalent Privacy (WEP) protocol was afforded solitary for the security over the wireless segment of the connection and it was able to afford end-to-end security [8].

\subsection{WiMAX (Worldwide Interoperability for Microwave Access):}

IEEE 802.16 is a progression of Wireless Broadband standards/prototypes. It is intended to build up standards for the worldwide exploitation of such broadband Wireless Metropolitan Area Networks (MAN). Despite the fact that it has been legitimately known as Wireless MAN in IEEE, but it has been commercialized by the name of "WiMAX" (Worldwide Interoperability for Microwave Access) by the industry agreement called the WiMAX Forum. Its goal is to bring wireless broadband services to distant access places devoid of hasty speed/pace cable or digital subscriber line (DSL) Internet service. Following are some traits of WiMAX:

i. It utilizes Orthogonal frequency division multiple access (OFDMA) and Orthogonal frequencydivision multiplexing (OFDM).

ii. WiMAX forum has been published three licensed spectrum: $2.3 \mathrm{GHz}, 2.5 \mathrm{GHz}$ and $3.5 \mathrm{GHz}$, in the unlicensed band, 5.x GHz is the permitted profile

iii. It endows with broadband wireless entrée up to 30 miles $(50 \mathrm{~km})$ for permanent stations, and $3-10$ miles $(5-15 \mathrm{~km})$ for mobile stations.

iv. It endows with an assortment of communication modes, from point-to-multipoint links to convenient and completely mobile internet access.

WiMAX is assumed to carry $70 \mathrm{Mbit} / \mathrm{s}$ over 30 miles or 50 $\mathrm{km}$; nevertheless, WiMAX is able to be operated either at privileged or higher bit rates or operated either over longer distances but no such capacity to be operated on both. In service over longer distances bit error rate enhances and in consequence outcome in a great deal of inferior bit rate. Additional the devise of the antennas also calls for the conciliation amid the bit rate and portability.

\subsection{ZigBee:}

It is a recognized set of stipulations for wireless personal area networking (WPAN based on the IEEE 802.15.4-2003 standards. This standard has been proposed by ZigBee Alliance. It is targeted at radio-frequency (RF) applications that entail a squat data rate, extensive battery existence, protected networking and trouble-free realization. Following are the most imperative aspects of ZigBee:

i. It employs three license-free bands: 2.4-2.4835 $\mathrm{GHz}, 868-870 \mathrm{MHz}$ and $902-928 \mathrm{MHz}$.

ii. It has utmost data rates permissible for each frequency bands are predetermined: $250 \mathrm{kbps}$ for $2.4 \mathrm{GHz}, 40 \mathrm{kbps}$ for $915 \mathrm{MHz}$, and $20 \mathrm{kbps}$ for 868 $\mathrm{MHz}$.

iii. Channel right to use utilizing Carrier Sense Multiple Access with Collision Avoidance (CSMA - CA).

iv. It has an address space of up to 64 bit IEEE address devices, bestow prospect of towering compactness of nodes per network.

v. It has a characteristic range of $50 \mathrm{~m}$.

\subsection{LoWPAN:}

6LoWPAN is a set of standards defined by the Internet Engineering Task Force (IETF), which constructs and sustains all core Internet architecture work and standards. 6LoWPAN standards make possible the efficient and resourceful utilization of IPv6 over low-power, low-rate wireless 
networks on simple embedded devices all the way through an adaptation layer and the optimization of related protocols. The IETF 6LoWPAN working group was legitimately founded in 2005, even though the history of embedded Internet Protocol goes back farther. All the way through in 1990s it was presupposed that Moore's law would progress computing and communication competence so quickly that in next to no time any embedded device may possibly put into practice the IP protocols. Even though to some extent true and the Internet of Things has matured quickly, it did not grasp for cheap, lowpower wireless radio technologies and low-power microcontrollers. A huge majority of simple embedded devices still employs 8-bit and 16-bit microcontrollers with very restricted memory, as they are low-power, cheap and small. Premature work on diminishing Internet protocols for bringing into play with low power microcontrollers and wireless technologies comprises NanoIP from the Centre for Wireless Communications [15] and $\mu \mathrm{IP}$ from the Swedish Institute of Computer Science [16]. IEEE 802.15.4 standard unveiled in 2003 and which was the major aspect leading to 6LoWPAN standardization. For the very first time a worldwide, extensively supported standard or model for lowpower wireless embedded communications was presented [17]. The esteem of this innovative standard gave the Internet society the required back-up to regulate an IP adaptation for such wireless embedded links. And consequently the first 6LoWPAN specifications were uncovered in 2007, first with an informational RFC [18] indicating the fundamental requirements and objectives of the primary standardization, and then with a standard track RFC [19] stipulating the 6LoWPAN format and its functionality. Some Important features of 6LoWPAN are:

$$
\begin{aligned}
& \text { v. It has a small device Networking. } \\
& \text { vi. As the future is an IP so it employs to carry data. } \\
& \text { vii. Its friendly for developers } \\
& \text { viii. IEEE 802.15.4 (Sub GHz or } 2.4 \mathrm{GHz} \text { - 6LoWPAN } \\
& \text { (RFC 6282) - IPv6 (RFC 2460) } \\
& \text { ix. RPL (RFC 6206-6550-6551) - CoAP (Drafts Core, } \\
& \text { Link Format, Blockwise and Observe) } \\
& \text { x. Application profile: IPSO or ZigBee® ZCL } \\
& \text { xi. Frequent interop tests with other vendors } \\
& \text { xii. Based on the Contiki open source operating system }
\end{aligned}
$$

\section{THE TECHNOLOGY OF WIRELESS COMMUNICATION:}

Standards similar to IrDA and Bluetooth deal with towering data rate applications such as voice, video and LAN interactions, while ZigBee marks small data rates, put away very small power and are therefore distinguished by extended battery existence. This turns out ZigBee to be a platform idyllic for the exclusive requirements of distant monitoring and control applications [10][11].

\section{Additional Wireless Technologies:}

A) The Ultra-Wideband (UWB Technology):

UWB technology for PAN puts forward an exceptional amalgamation of low power utilization.

$\sim 1 \mathrm{~mW} / \mathrm{Mbps}$ and towering data throughput up to $480 \mathrm{Mbps}$. WiMedia Ultra-Wideband is a globally acknowledged standard/prototype (ECMA-368, ISO/IEC 26970 and ECMA369 , ISO/IEC 26908) and has authoritarian endorsement in most important markets all-inclusive. WiMedia Ultra-
Wideband consent ranges of more than a few meters and a data rate of roughly $110 \mathrm{Mbps}$ at a range of up to 10 meters [12].

\section{B) The (RFID's) Technology:}

The term RFID stands for Radio Frequency Identification. At the moment we have more than 140 diverse ISO standards/prototypes for RFID for an extensive range of applications. By means of RFID, a reader device can power an unreceptive or unpowered tag. The recipient, which be obliged to be inside a few feet, drags information off the 'tag,' and subsequently look up more information from a database. On the other hand, a number of tags are self-powered, 'active' tags that can be interpret from a larger distance RFID operates at diverse frequencies ranges, together with $125 \mathrm{KHz}, 13.56$ $\mathrm{MHz}, 2.45 \mathrm{GHz}$ and $5.8 \mathrm{GHz}$, and $860-950 \mathrm{MHz}$. bestows read ranges from a small number of centimeters to 5 meters, depending on the frequency of the devices. [13]

\section{AN EMBEDDED SYSTEMS BASED ASSESSMENT FOR TECHNOLOGIES:}

Outlay, size, power and performance are the frequent devise metrics/dimensions or parameters ought to be well thoughtout for any computing system. And such these parameters or metrics should be obligatory and tight in case of embedded. Embedded system frequently have got to be pricing smallest, be obliged to be sized to in shape on a single chip or a board, ought to be imprudent to the surroundings, effort prompt as much as necessary to practice data in real time, and be obliged to put away bare minimum power to make longer battery existence. Both Wi-Fi and WiMAX technology are intended for longer distance and privileged bit rate connections and devours more energy [14]. In view of this fact, Bluetooth, IrDA, ZigBee, 6LowPAN are superior substitutes for shortrange wireless transmission in embedded systems. On the other hand, each technology has their pros and cons and don't have the aptitude to assemble requirements of each system. Characteristics of each can be evaluated as follows:

Bluetooth vs. IrDA: Bluetooth is a Radio technology and has the capability to infiltrate unyielding objects and its qualifications for utmost mobility surrounded by the piconet tolerate data exchange/swapping applications that are problematic or unachievable with IrDA. In contrast, as in IrDA communication the signal is not able to penetrate obscure objects, as a result crafting eavesdrop/snooping difficult and dipping intrusion. Additional, infrared links are not as vulnerable to multi-path fading as radio links, for the reason that of the diminutive carrier wavelength. Bluetooth transmissions are Omni-directional; as a result there are no line-of-sight concerns as in IrDA when communication comes in being. Bluetooth endows with vigorous safety measures methods as compared to IrDA where it is missing. In contrast, IrDA put away less power measure up to Bluetooth and are simpler to put into practice and by and large comes out to be inexpensive put side by side to Bluetooth elucidations.

Bluetooth vs. ZigBee: Both are Radio technology. Nevertheless, both are beleaguered for unusual application fields. Bluetooth is destined for areas like PDAs handsets, wireless USB's etc, while, ZigBee is doomed to accommodate to the remote control market and sensors and other battery operated stuffs. Bandwidth of Bluetooth is pretty towering in contrast to ZigBee, Regarding the stack size so protocol stack size of ZigBee is moderately fewer than that of Bluetooth stack size. Interoperability, flexibility and substitution of cables are the potency of Bluetooth. Whereas in case of 
ZigBee's so is cheaper, inexpensive, and extensive battery existence.

\section{Zigbee vs. 6lowPan:}

ZigBee is a minute-scale one-off ad-hoc networking whereas 6LoWPAN is particularly scalable networking as an end-toend fraction of the Internet, it is IPv6. ZigBee is restricted to a solitary radio standard whereas 6LoWPAN is pertinent to any low-rate, low-power wireless radio or even wired. IP protocols bind together diverse networks. The barely fine element of ZigBee is application protocol profiles. And presume what, there is an IETF measurement for utilizing ZigBee profiles above UDP/IP. ZigBee is not a standard rather than a meticulous interest group. Will it be something like in a small number of years? The IETF constructs open, extensive subsisted standards. IPv6 will be in the region for 20 and more years. M2M, Large-scale enterprise automation, metering systems etc. entail end-to-end addressing, mobility, security, traffic multiplexing, maintainability, reusability and web-services which are worldwide scalable. And for such variety and purpose IPV6 was intended.

\section{SUMMARY:}

Currently we have an assortment of novel technologies on hand for restricted interaction or communication such as Bluetooth, IrDA, Wi-Fi, WiMAX, ZigBee and 6LowPan etc. All have been examined and some of them are under consideration or under study. A number of aspects make your mind up about the aptness of wireless technology for an application is well thought-out. We outlined most trendy wireless technologies namely Bluetooth, IrDA and ZigBee, 6LowPAN in provisions of their characteristics, Pros and Cons. By now as we know that the areas of deployment for such embedded systems are so unusual and diverse, so in view of this fact, their selection and implementation of a well suited embedded system technology will be purely based on the nature, sort and size of the applications.

\section{REFERENCES:}

[1] "Jonathan and Valveno", "Embedded Microcomputer Systems", Eastern Press, 2005.

[2] "Ganssle", "Art of Designing Embedded System", Newness pub. ltd., 2004.

[3] "M2M Telematics Ltd. (2007)", "A white paper on the benefits of remote access technology"

[4] "D. Agrawal and R. Malladi" "Current and future applications of mobile and wireless networks," Communications of the ACM, vol. 45, no. 10, pp. 144146, 2002.
[5] "Bluetooth SIG", Inc. (2009), Bluetooth Basics

[6] [6] "Agilent Technologies, Inc. (2006)", Bluetooth Enhanced Data Rate (EDR): The Wireless Evolution Application Note"

[7] 'IrDA, (2009)", "IrDA Background". IrDA

[8] "IEEE Standard Association (2009)", "IEEE 802.11: Wireless LAN Medium Access Control (MAC) and Physical Layer (PHY) Specifications"

[9] "IEEE Standard Association (2009)", “IEEE 802.16 Broadband Wireless Metropolitan Area Network Specifications".

[10] ZigBee Alliance (2009)", ZigBee Technical document

[11] "Daintree Networks (2009), Introducing ZigBee RF4CE

[12] "ECMA International (2009), Standard ECMA-368 High Rate Ultra Wideband PHY and MAC Standard".

[13] Alien Technology (2009), About RFID” http://www.alientechnology.com/

[14] Socket Communications, Inc (2009), Bluetooth and WiFi: Understanding these two technologies and how they can benefit you".

[15] Shelby, Z., Mähönen, P., Riihijärvi, J.O.R. and Huuskonen, P. NanoIP: The Zen of Embedded Networking. In Proceedings of the IEEE International Conference on Communications. May 2003.

[16] Dunkels, A. Full TCP/IP for 8-Bit Architectures. In Proceedings of the First ACM/Usenix International Conference on Mobile Systems, Applications and Services (MobiSys 2003). ACM, San Francisco, May 2003.

[17] IEEE Std 802.15.4TM-2006: Wireless Medium Access Control (MAC) and Physical Layer (PHY) Specifications for Low-Rate Wireless Personal Area Networks (LR WPANs), October 2006.

[18] Kushalnagar, N., Montenegro, G. and Schumacher, C. IPv6 over Low-Power WirelessPersonal Area Networks (6LoWPANs): Overview, Assumptions, Problem Statement, and Goals. RFC 4919, Internet Engineering Task Force, Aug. 2007.

[19] Montenegro, G., Kushalnagar, N., Hui, J. and Culler, D. Transmission of IPv6 Packets over IEEE 802.15.4 Networks. RFC 4944, Internet Engineering Task Force, Sep. 2007. 